\title{
Deficiências de macronutrientes no estado nutricional da mamoneira cultivar Iris
}

\author{
José Lavres Junior(1), Rodrigo Marcelli Boaretto(1), Maria Lígia de Souza Silva(2), Diva Correia ${ }^{(3)}$, \\ Cleusa Pereira Cabral ${ }^{(1)}$ e Eurípedes Malavolta ${ }^{(1)}$
}

\begin{abstract}
(1)Universidade de São Paulo (USP), Centro de Energia Nuclear na Agricultura (CENA), Caixa Postal 96, CEP 13400-970 Piracicaba, SP. E-mail: jlavres@cena.usp.br, rmboaret@cena.usp.br, cpcabral@cena.usp.br, mala@cena.usp.br (2)USP, Escola Superior de Agricultura Luiz de Queiroz, Dep. de Solos e Nutrição de Plantas, Caixa Postal 9, CEP 13418-900 Piracicaba, SP. E-mail: mlssilva@esalq.usp.br (3)Embrapa Agroindústria Tropical, Caixa Postal 3761, CEP 60511-110 Fortaleza, CE. E-mail: diva@cnpat.embrapa.br
\end{abstract}

\begin{abstract}
Resumo - Os objetivos deste trabalho foram avaliar o efeito da deficiência dos macronutrientes no crescimento e estado nutricional da mamoneira (Ricinus communis L.) e a obtenção do quadro sintomatológico das deficiências de N, P, K, Ca, Mg e S. Cultivou-se Ricinus communis L. cultivar Iris em solução nutritiva, tendo como tratamentos soluções completa e deficientes em N, P, K, Ca, Mg e S. Foram determinadas a concentração indireta de clorofila, a atividade da redutase do nitrato, os teores solúveis de $\mathrm{N}\left(\mathrm{NO}_{3}^{-}\right), \mathrm{P}\left(\mathrm{H}_{2} \mathrm{PO}_{4}^{-}\right)$e $\mathrm{K}^{+}$e realizados testes rápidos para $\mathrm{N}, \mathrm{P}$ e $\mathrm{K}$. As deficiências de $\mathrm{N}, \mathrm{Ca}, \mathrm{S}$ e $\mathrm{Mg}$ foram as que mais restringiram a produção de massa de matéria seca, na ordem decrescente: $\mathrm{N}>\mathrm{Ca}>\mathrm{S}>\mathrm{Mg}>\mathrm{K}>\mathrm{P}$. Os maiores acúmulos de macronutrientes, no tratamento completo, foram observados nas raízes e no limbo das folhas inferiores. As concentrações críticas para $\mathrm{Ne} \mathrm{Ca}$, ambas no limbo das folhas superiores e, $\mathrm{Mg}$, no pecíolo das inferiores foram, respectivamente, 46,7, 13,8 e $6,5 \mathrm{~g} \mathrm{~kg}^{-1}$. As deficiências influenciaram as leituras do clorofilômetro. A atividade da redutase do nitrato guardou relação direta com a determinação da clorofila. Os teores de $\mathrm{N}\left(\mathrm{NO}_{3}^{-}\right), \mathrm{P}_{(}\left(\mathrm{H}_{2} \mathrm{PO}_{4}^{-}\right)$e $\mathrm{K}^{+}$foram menores nas folhas deficientes em N, P e K, respectivamente, o mesmo acontecendo quando avaliadas pela análise de toque.
\end{abstract}

Termos para indexação: Ricinus communis, clorofilômetro, nutrição, sintomas.

\section{Deficiencies of macronutrients on nutritional status of castor bean cultivar Iris}

\begin{abstract}
The objectives of this work were to evaluate the effect of macronutrient deficiencies on growth and on nutritional status of castor beans (Ricinus communis L.), and to obtain the symptoms of the lack of N, P, K, Ca, $\mathrm{Mg}$ and $\mathrm{S}$. Nutrient solutions containing all macronutrients and those from which one of these elements was omitted were the treatments used. The following determinations were made: chlorophyll (indirectly), nitrate reductase activity, $\mathrm{NO}_{3}{ }^{-}-\mathrm{N}, \mathrm{H}_{2} \mathrm{PO}_{4}^{-}-\mathrm{P}$ and $\mathrm{K}^{+}$, both on soluble fractions, and by spot tests. Deficiencies of $\mathrm{N}, \mathrm{Ca}$, $\mathrm{S}$ and $\mathrm{Mg}$ were the most limiting for dry matter production, followed in decreasing order by those of $\mathrm{K}$ and $\mathrm{P}$. The highest concentrations of macronutrients were measured in roots and lower leaf blades. Critical levels of $\mathrm{N}$ and $\mathrm{Ca}$ in the upper leaf blades and of $\mathrm{Mg}$ in lower petioles were respectively, 46.7, 13.8, $6.5 \mathrm{~g} \mathrm{~kg}^{-1}$. Deficiencies affected chlorophyll meter readings. Nitrate reductase activity was correlated with chlorophyll readings. Soluble contents of N, P and $\mathrm{K}$ in the leaves were lower in plants deficient in the respective element. The same was found when spot tests were applied on the leaf sap.
\end{abstract}

Index terms: Ricinus communis, chlorophyll meter, nutrition, symptoms.

\section{Introdução}

A mamoneira (Ricinus communis L.) pertence à família Euphorbiaceae, possivelmente originária da antiga Abissínia, hoje Etiópia (Beltrão et al., 2001). Do ponto de vista agroindustrial, o fruto apresenta aproveitamento integral, obtendo-se como produto principal o óleo e, como subproduto, a torta que pode ser utilizada como adubo orgânico (Beltrão et al., 2001). A ricinoquímica é responsável pela produção de mais de 400 subprodutos, tais como, plásticos, fibras sintéticas, tintas, esmaltes, lubrificantes e combustível, além de servir de matériaprima na produção de próteses em substituição à platina, com custo reduzido, cimento ósseo, ação fungicida e bactericida (Freire, 2001).

O Brasil é o terceiro maior produtor mundial, com previsão de área plantada de 127.688 ha no ano agrícola de 2003. No ano de 2001, em 176.534 ha, foram produzidas 99.950 toneladas de mamona em baga (IBGE, 2003). No país, as áreas cultivadas com mamona têm 
crescido, sendo também explorada no Cerrado das Regiões Nordeste e Centro-Oeste, visando principalmente à produção do biodiesel (Beltrão et al., 2002). Adicionalmente, na Região Centro-Oeste, a mamoneira tem sido utilizada como cultura destinada à rotação com o cultivo da soja, diversificando, deste modo, a fonte de renda dos produtores.

Vários estudos sobre adubação em mamoneira com N, P e K foram realizados (Canecchio Filho \& Freire, 1958; Canecchio Filho et al., 1963; Rocha et al., 1964; Nakagawa et al., 1982). Entretanto, poucos foram direcionados à avaliação do estado nutricional da cultura, fornecendo indicações das suas exigências (Rojas \& Neptune, 1971; Hocking, 1982).

Os objetivos deste trabalho foram avaliar o efeito da deficiência dos macronutrientes no crescimento e estado nutricional da mamoneira (Ricinus communis L.) cultivar Iris, e a obtenção do quadro sintomatológico das deficiências de N, P, K, Ca, Mg e S.

\section{Material e Métodos}

O experimento foi realizado em casa de vegetação, no Centro de Energia Nuclear na Agricultura - USP, em Piracicaba, SP, no período de fevereiro a junho de 2003. Foram utilizadas plantas de mamoneira (Ricinus communis L.) do híbrido comercial Iris, selecionado para condições de Cerrado, cujas características principais são: porte baixo, precocidade, não-deiscência e adequação à colheita mecânica.

As sementes foram colocadas para germinar em bandeja rasa com vermiculita, umedecida com solução de sulfato de cálcio $\left(\mathrm{CaSO}_{4}, 10^{-4} \mathrm{~mol} \mathrm{~L}^{-1}\right)$. Quando as plantas atingiram cerca de $5 \mathrm{~cm}$ de altura, foram transferidas para bandeja de plástico com capacidade de $40 \mathrm{~L}$ contendo solução nutritiva (Johnson et al., 1957) completa e diluída a $1 / 5$ da concentração usual. As plantas foram fixadas na região do colo com espuma de plástico.

Aos 21 dias após emergência, iniciaram-se os tratamentos, utilizando-se uma planta por vaso de $1,5 \mathrm{~L}$ revestidos com papel-alumínio. Durante a condução do experimento, as soluções nutritivas, sob constante aeração, foram renovadas a cada 15 dias, completando-se o volume com água desmineralizada, quando necessário. $\mathrm{O} \mathrm{pH}$ foi mantido em torno de 5,5.

O delineamento experimental utilizado foi inteiramente casualizado com os tratamentos arranjados nas unidades experimentais em parcelas subdivididas, constando de seis repetições para os tratamentos completo e deficientes em N, P e K, e três repetições para os tratamentos deficientes em $\mathrm{Ca}, \mathrm{Mg}$ e $\mathrm{S}$, em que as parcelas corresponderam a esses tratamentos e as subparcelas às épocas de avaliações (avaliações semanais).

Semanalmente, efetuou-se a determinação indireta do teor de clorofila expresso em valores SPAD, com uso do Chlorophyll Meter SPAD-502 (Minolta Camera Co., 1989), no lóbulo mediano da terceira folha superior recém-madura.

Aos 91 dias após início dos tratamentos, as plantas foram colhidas e separadas em caule, folhas inferiores e superiores (limbo e pecíolo) e raízes. O material foi identificado, acondicionado em sacos de papel e secado em estufa a $65^{\circ} \mathrm{C}$ durante 72 horas. Posteriormente foi pesado, triturado em moinho tipo Wiley (peneira com diâmetro de malha de $1 \mathrm{~mm}$ ) e realizadas as determinações químicas de $\mathrm{N}, \mathrm{P}, \mathrm{K}, \mathrm{Ca}, \mathrm{Mg}$ e $\mathrm{S}$ nos tecidos segundo Malavolta et al. (1997).

Aos 35 dias após o início dos tratamentos, efetuou-se a determinação indireta da concentração de clorofila nos lóbulos medianos da quarta folha, a partir do ápice, nas plantas correspondentes aos tratamentos completo e com deficiência em nitrogênio. Em seguida usou-se essa folha na determinação da atividade da redutase do nitrato, conforme adaptação do método descrito por Mulder et al. (1959). Foram determinados os teores solúveis de $\mathrm{N}$ $\left(\mathrm{NO}_{3}{ }^{-}\right), \mathrm{P}\left(\mathrm{H}_{2} \mathrm{PO}_{4}^{-}\right)$e $\mathrm{K}^{+}$(Ulrich, 1948) e feitos os testes rápidos (Fernandes, 1967; Malavolta, 1975), ambos no pecíolo da folha superior, no tratamento completo e nos tratamentos com deficiência em N, P e K.

Adotou-se o critério estabelecido por Ulrich \& Hill (1973), os quais definiram o nível crítico como a concentração do nutriente que corresponde a $90 \%$ da produção máxima da cultura. Dessa forma, determinou-se a concentração crítica dos macronutrientes nas partes da planta que apresentaram maior coeficiente de correlação (r) e significância para a relação.

Os dados foram submetidos às análises estatísticas utilizando-se o programa estatístico SAS - System for Windows 6.11 (SAS Institute, 1996). Realizou-se a análise de variância e em função do nível de significância pelo teste $\mathrm{F}$, para o efeito dos tratamentos e das épocas de avaliação, procedeu-se ao estudo de regressão dos componentes de primeiro e segundo grau, mediante procedimento GLM e o teste de Tukey a 5\% de probabilidade na comparação das médias entre os tratamentos. 
As correlações entre as variáveis concentração indireta de clorofila e atividade enzimática da redutase do nitrato foram obtidas e testadas mediante procedimento CORR, pelo programa estatístico SAS - System for Windows 6.11 (SAS Institute, 1996).

\section{Resultados e Discussão}

Os sintomas visuais correspondem às deficiências mais acentuadas dos nutrientes na última semana de avaliação do experimento (Figura 1). Os primeiros sintomas visuais de deficiência ocorreram nas folhas velhas em plantas mantidas em solução deficiente em N aos 21 dias após o início dos tratamentos. Em seguida ocorreu a deficiência de $\mathrm{K}$, também em folhas velhas e aos 28 dias. Os sintomas de carência de $\mathrm{Ca}$ (folhas novas), $\mathrm{Mg}$ (folhas velhas) e S (folhas novas) manifestaram-se aos 49 dias, e os de falta de $\mathrm{P}$ (folhas velhas), após 80 dias. Os sintomas manifestados foram semelhantes aos descritos por Rojas \& Neptune (1971), na cultivar Campinas.

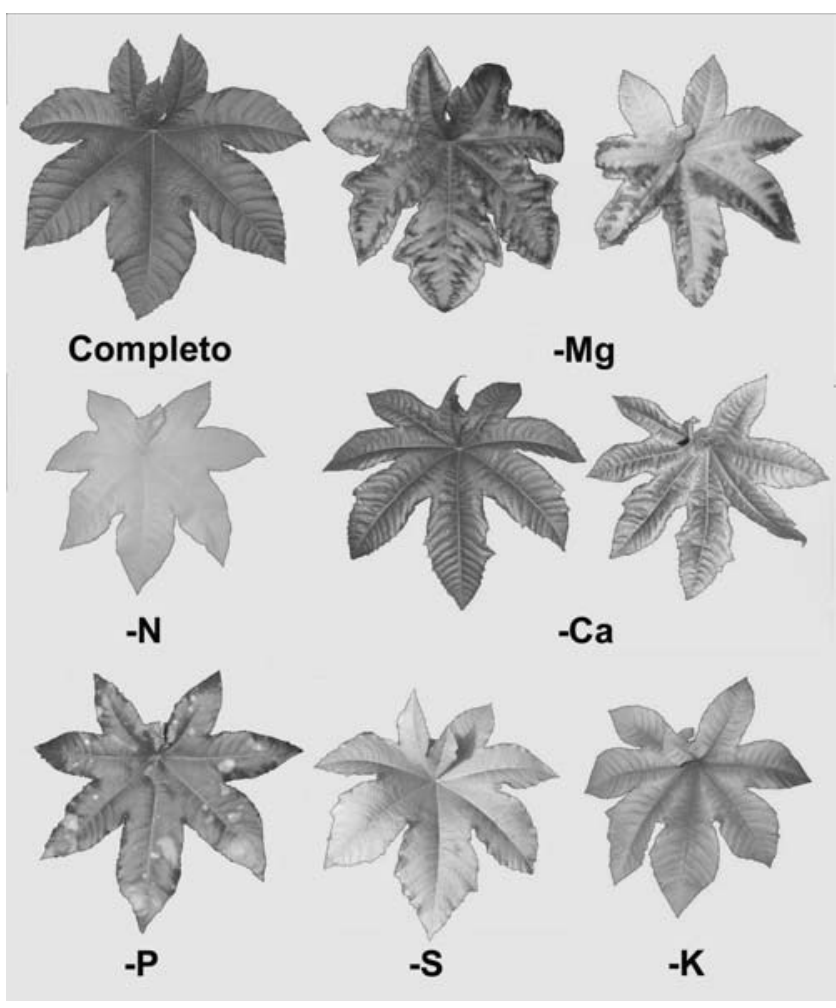

Figura 1. Sintomas das deficiências na mamoneira cv. Iris, desenvolvida em solução nutritiva completa e com deficiências em N, P, K, Ca, Mg e S.
A deficiência de $\mathrm{N}$ ocasionou redução na concentração deste nutriente apenas em relação à sua concentração na raiz e pecíolo superior (Tabela 1). No tratamento completo, a maior concentração ocorreu no limbo superior, o que indica alta mobilidade do elemento na planta (Epstein, 1975).

No tratamento deficiente em $\mathrm{P}$ ocorreu diminuição do seu teor nas partes da planta. No tratamento completo, a maior concentração de $\mathrm{P}$ foi observada nas raízes enquanto no tratamento deficiente em P, no limbo superior, conseqüência de redistribuição (Mengel \& Kirkby, 2001).

$\mathrm{O}$ teor de $\mathrm{K}$ diminuiu em todas as partes da planta com a deficiência deste nutriente. No tratamento completo, houve maior concentração de $\mathrm{K}$ no limbo inferior, enquanto no tratamento com deficiência, a maior concentração ocorreu no limbo superior, o que pode ser explicado pela elevada mobilidade do K na planta, quanto à redistribuição (Epstein, 1975; Mengel \& Kirkby, 2001).

Os teores solúveis encontrados nas plantas das soluções completa e deficientes em $\mathrm{N}, \mathrm{P}$ e K foram 0,5 e $0,2 \mathrm{~g} \mathrm{~kg}^{-1} \mathrm{de} \mathrm{N}\left(\mathrm{NO}_{3}^{-}\right) ; 2,0$ e $0,5 \mathrm{~g} \mathrm{~kg}^{-1}$ de $\mathrm{P}\left(\mathrm{H}_{2} \mathrm{PO}_{4}^{-}\right)$; e 17,3 e $3,5 \mathrm{~g} \mathrm{~kg}^{-1} \mathrm{de} \mathrm{K}^{+}$, respectivamente. Estes teores concordam com a avaliação efetuada pelo teste rápido de N, P e K também realizado no pecíolo superior das folhas durante o período de crescimento, e com os resultados obtidos na análise química de tecido vegetal realizada no final do experimento (Tabela 1).

Com relação ao $\mathrm{Ca}$, constatou-se redução na concentração nas partes da planta quando em deficiência, comparado ao tratamento completo. Tanto no tratamento completo quanto no tratamento com deficiência em $\mathrm{Ca}$, o maior teor do nutriente ocorreu no limbo inferior, indicação da baixa mobilidade desse nutriente na planta (Epstein, 1975; Mengel \& Kirkby, 2001).

Os teores de $\mathrm{Mg}$, nas plantas do tratamento com deficiência, foram inferiores aos do completo, no pecíolo superior, no limbo e pecíolo inferiores. Porém, no tratamento deficiente em $\mathrm{Mg}$ houve maior concentração deste elemento no caule e nas raízes, diferindo significativamente das demais partes. $\mathrm{O}$ mesmo não pode ser observado no tratamento completo, no qual os teores nas partes da planta foram semelhantes, diferindo significativamente apenas no caule. 
As concentrações de $\mathrm{S}$ nas partes da planta, no tratamento com deficiência foram menores quando comparadas com o tratamento completo, apenas no limbo da folha inferior. Em ambos os tratamentos, o limbo da folha inferior apresentou maior concentração de S, elemento de baixa mobilidade (Epstein, 1975; Mengel \& Kirkby, 2001).

As concentrações de $\mathrm{K}$, nos tratamentos deficientes em $\mathrm{K}$ e $\mathrm{Ca}$, diferenciaram-se estatisticamente apenas em relação ao limbo e pecíolo inferiores, enquanto que em relação às concentrações de $\mathrm{Ca}$, nas diferentes partes, constataram-se diferenças significativas, em ambos tratamentos, exceto no pecíolo superior (Tabela 1). O mesmo efeito também foi verificado nas concentrações de $\mathrm{Mg}$, nos tratamentos deficientes em $\mathrm{K}$ e Ca, exceto nos limbos superiores e inferiores. Porém, de maneira geral, no tratamento com deficiência de $\mathrm{K}$, as concentrações de $\mathrm{Mg}$ no tecido vegetal foram superiores às do tratamento com deficiência em $\mathrm{Ca}$, em concordância com as observações de Veloso et al. (1998) que, na condição de omissão de K, na solução nutritiva, relataram aumento do teor de $\mathrm{Mg}$ nas partes da pimenta-do-reino. Os maiores teores foram encontrados nas omissões de $\mathrm{K}$ e Ca, caracterizando o efeito da inibição competitiva do K na absorção do Mg (Mengel \& Kirkby, 2001).

No tratamento com deficiência em $\mathrm{K}$, as maiores concentrações de $\mathrm{K}$, Ca e Mg foram observadas, respectivamente, no limbo superior, no limbo inferior e pecíolo superior (Tabela 1). No tratamento com deficiência em $\mathrm{Ca}$, as maiores concentrações de $\mathrm{K}, \mathrm{Ca}$ e $\mathrm{Mg}$ corresponderam ao pecíolo inferior, limbo inferior e pecíolo inferior, respectivamente. Segundo Malavolta et al. (1997), altas concentrações de Ca, e principalmente

Tabela 1. Concentração $\left(\mathrm{g} \mathrm{kg}^{-1}\right)$ dos macronutrientes na mamoneira, cv. Iris, desenvolvida em solução nutritiva completa e com deficiência em N (Def-N), P (Def-P), K (Def-K), Ca (Def-Ca), Mg (Def-Mg) e S (Def-S), e concentração de K, Ca e Mg na mamoneira desenvolvida em soluções nutritivas deficientes em $\mathrm{Ke} \mathrm{Ca}^{(1)}$.

\begin{tabular}{|c|c|c|c|c|c|c|}
\hline Parte da planta & Completo & Def-N & Completo & Def-P & Completo & Def-K \\
\hline & \multicolumn{2}{|c|}{$\mathrm{N}$} & \multicolumn{2}{|c|}{$\mathrm{P}$} & \multicolumn{2}{|c|}{$\mathrm{K}$} \\
\hline Limbo superior & $48,1 \mathrm{aA}$ & $30,0 \mathrm{aA}$ & $4,7 \mathrm{cA}$ & $3,5 \mathrm{aB}$ & $23,3 \mathrm{bA}$ & $14,3 \mathrm{aA}$ \\
\hline Pecíolo superior & $25,1 \mathrm{bcA}$ & $12,0 \mathrm{bcB}$ & $6,5 \mathrm{bA}$ & $1,9 \mathrm{abB}$ & $26,5 \mathrm{abA}$ & $8,8 \mathrm{bB}$ \\
\hline Limbo inferior & $26,9 \mathrm{bcA}$ & $17,7 \mathrm{bA}$ & $4,3 \mathrm{cA}$ & $2,4 \mathrm{abA}$ & $30,1 \mathrm{aA}$ & $9,4 \mathrm{bB}$ \\
\hline Pecíolo inferior & $19,9 \mathrm{cdA}$ & $9,5 \mathrm{cA}$ & $4,5 \mathrm{cA}$ & $1,1 \mathrm{bB}$ & $24,6 \mathrm{abA}$ & $3,8 \mathrm{cB}$ \\
\hline Caule & $13,3 \mathrm{dA}$ & $8,5 \mathrm{cA}$ & $2,5 \mathrm{dA}$ & $1,0 \mathrm{bB}$ & $10,9 \mathrm{cA}$ & $3,0 \mathrm{cB}$ \\
\hline Raiz & $32,7 \mathrm{bA}$ & $12,9 \mathrm{bcB}$ & $8,0 \mathrm{aA}$ & $1,6 \mathrm{abB}$ & $27,2 \mathrm{abA}$ & $6,6 \mathrm{bcB}$ \\
\hline \multirow[t]{3}{*}{$\mathrm{CV}(\%)$} & 10,85 & 18,10 & 4,17 & 38,00 & 8,77 & 18,51 \\
\hline & Completo & Def-Ca & Completo & Def-Mg & Completo & Def-S \\
\hline & \multicolumn{2}{|c|}{$\mathrm{Ca}$} & \multicolumn{2}{|c|}{$\mathrm{Mg}$} & \multicolumn{2}{|c|}{$\mathrm{S}$} \\
\hline Limbo superior & $13,6 \mathrm{aA}$ & $1,1 \mathrm{bB}$ & $6,7 \mathrm{abA}$ & $4,2 \mathrm{bA}$ & $3,7 \mathrm{bA}$ & $2,6 \mathrm{abcA}$ \\
\hline Pecíolo superior & $7,7 \mathrm{aA}$ & $1,7 \mathrm{bB}$ & $8,8 \mathrm{aA}$ & $1,9 \mathrm{cB}$ & $1,7 \mathrm{cA}$ & $1,7 \mathrm{cdA}$ \\
\hline Limbo inferior & $15,5 \mathrm{aA}$ & $4,6 \mathrm{aA}$ & $8,0 \mathrm{aA}$ & $3,0 \mathrm{bcB}$ & $4,4 \mathrm{aA}$ & $3,4 \mathrm{aB}$ \\
\hline Pecíolo inferior & $10,8 \mathrm{aA}$ & $4,1 \mathrm{aA}$ & 7,1abA & $2,1 \mathrm{cB}$ & $1,3 \mathrm{cdA}$ & $1,5 \mathrm{bcdA}$ \\
\hline Caule & $7,5 \mathrm{a}$ & $-(2)$ & $4,3 \mathrm{bA}$ & $7,8 \mathrm{aA}$ & $0,8 \mathrm{dA}$ & $0,6 \mathrm{dA}$ \\
\hline$\underline{\text { Raiz }}$ & $8,1 \mathrm{aA}$ & $1,1 \mathrm{bB}$ & $6,6 \mathrm{abA}$ & $6,9 \mathrm{aA}$ & $3,0 \mathrm{bA}$ & 3,3abA \\
\hline \multirow[t]{3}{*}{$\mathrm{CV}(\%)$} & 31,0 & 26,24 & 17,83 & 11,11 & 12,50 & 32,90 \\
\hline & \multicolumn{3}{|c|}{ Tratamento deficiente em $\mathrm{K}$} & \multicolumn{3}{|c|}{ Tratamento deficiente em $\mathrm{Ca}$} \\
\hline & $\mathrm{K}$ & $\mathrm{Ca}$ & $\mathrm{Mg}$ & $\mathrm{K}$ & $\mathrm{Ca}$ & $\mathrm{Mg}$ \\
\hline Limbo superior & $14,3 \mathrm{aA}$ & $5,8 \mathrm{cdA}$ & $6,4 \mathrm{bcA}$ & $21,1 \mathrm{bcA}$ & $1,1 \mathrm{aB}$ & $5,1 \mathrm{abA}$ \\
\hline Pecíolo superior & $8,8 \mathrm{bA}$ & $7,3 \mathrm{cdA}$ & $12,1 \mathrm{aA}$ & $10,8 \mathrm{cA}$ & $2,9 \mathrm{aA}$ & $3,8 \mathrm{bB}$ \\
\hline Limbo inferior & $9,4 \mathrm{bB}$ & $19,6 \mathrm{aA}$ & $10,3 \mathrm{aA}$ & $25,8 \mathrm{abA}$ & $4,6 \mathrm{aB}$ & $7,5 \mathrm{abA}$ \\
\hline Pecíolo inferior & $3,8 \mathrm{cB}$ & $13,9 \mathrm{bA}$ & $11,2 \mathrm{aA}$ & $33,2 \mathrm{aA}$ & $4,1 \mathrm{aB}$ & $9,4 \mathrm{aB}$ \\
\hline Caule & $3,0 \mathrm{c}$ & $9,7 b c$ & $4,6 \mathrm{c}$ & $-(2)$ & $-(2)$ & $-(2)$ \\
\hline Raiz & $6,6 \mathrm{bcA}$ & $5,1 \mathrm{dA}$ & $7,0 \mathrm{bA}$ & $17,1 \mathrm{bcA}$ & $1,1 \mathrm{aB}$ & $4,4 \mathrm{bB}$ \\
\hline$\overline{\mathrm{CV}(\%)}$ & 18,51 & 14,70 & 9,21 & 17,99 & 47,68 & 27,91 \\
\hline
\end{tabular}

(1)Médias seguidas por letras distintas, minúsculas na coluna e maiúsculas na linha, diferem entre si pelo teste de Tukey a 5\% de probabilidade. (2)Dados não mostrados. 
Tabela 2. Quantidade acumulada dos macronutrientes $\left(\mathrm{mg} \mathrm{planta}^{-1}\right)$ na mamoneira, cv. Iris, desenvolvida em solução nutritiva completa $^{(1)}$.

\begin{tabular}{|c|c|c|c|c|c|c|}
\hline Parte da planta & $\mathrm{N}$ & $\mathrm{P}$ & K & $\mathrm{Ca}$ & $\mathrm{Mg}$ & S \\
\hline Limbo superior & $112 \mathrm{bc}$ & $11 \mathrm{c}$ & $55 \mathrm{~cd}$ & $32 b$ & $16 \mathrm{~cd}$ & $8 b$ \\
\hline Pecíolo superior & $18 \mathrm{c}$ & $3 c$ & $11 \mathrm{~d}$ & $3 b$ & $4 d$ & $1 \mathrm{c}$ \\
\hline Limbo inferior & $179 b$ & $29 b$ & $199 b$ & $105 a$ & $52 \mathrm{ab}$ & $29 a$ \\
\hline Pecíolo inferior & $32 \mathrm{c}$ & $7 c$ & $43 \mathrm{~cd}$ & $22 b$ & $12 \mathrm{~cd}$ & $2 b c$ \\
\hline Caule & $98 \mathrm{bc}$ & $18 \mathrm{bc}$ & $81 \mathrm{c}$ & $56 a b$ & $33 \mathrm{bc}$ & $6 b c$ \\
\hline Raiz & $373 a$ & $90 \mathrm{a}$ & $309 a$ & $91 \mathrm{a}$ & $76 a$ & $34 \mathrm{a}$ \\
\hline Total & 812 & 158 & 698 & 309 & 193 & 80 \\
\hline $\mathrm{CV}(\%)$ & 25,33 & 21,24 & 19,94 & 38,78 & 31,91 & 16,20 \\
\hline
\end{tabular}

${ }^{(1)}$ Médias seguidas por letras distintas, na coluna, diferem entre si pelo teste de Tukey a $5 \%$ de probabilidade.
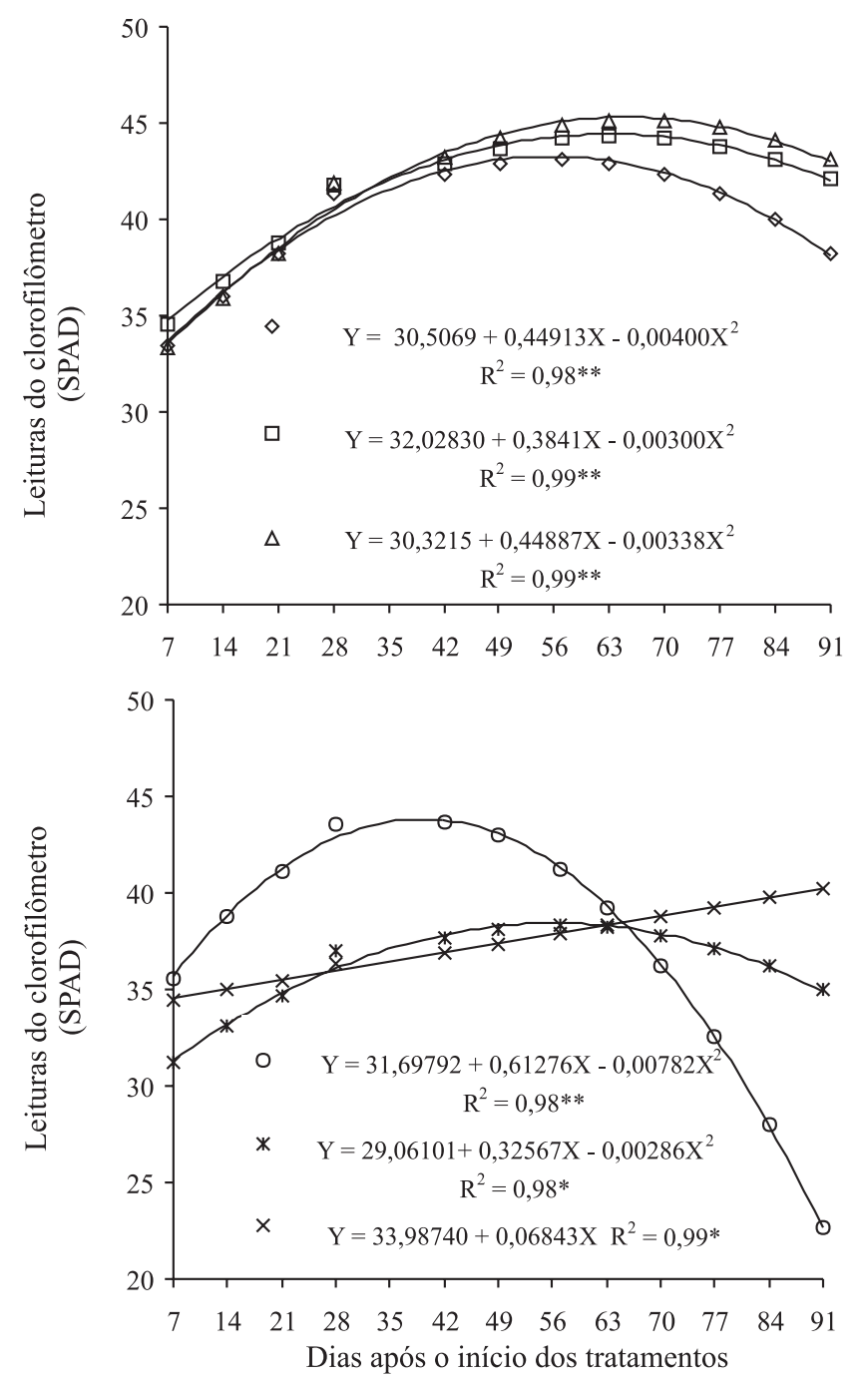

Figura 2. Determinação indireta do teor de clorofila, em valores SPAD, na mamoneira cv. Íris desenvolvida em solução nutritiva completa $(\diamond)$ e com deficiência em $\mathrm{P}(\square), \mathrm{K}(\triangle)$, $\mathrm{Ca}(\mathrm{O}), \mathrm{Mg}(*)$ e $\mathrm{S}(\times)$, em função dos dias após o início dos tratamentos. $* \mathrm{e}^{* *}$ Significativo a $5 \%$ e a $1 \%$ de probabilidade, respectivamente, pelo teste $\mathrm{F}$. de $\mathrm{K}$ no meio, podem inibir competitivamente a absorção causando, às vezes, a deficiência mais comumente observada em culturas exigentes em potássio.

Quanto às quantidades acumuladas dos macronutrientes nas partes da planta, no tratamento completo, as raízes foram as que apresentaram maior acúmulo, seguidas do limbo inferior, exceto para o $\mathrm{Ca}$ que apresentou comportamento inverso, ou seja, o acúmulo foi maior no limbo inferior seguido pelas raízes (Tabela 2). A acumulação de nutrientes obedeceu à seguinte ordem decrescente $\mathrm{N}>\mathrm{K}>\mathrm{Ca}>\mathrm{Mg}>\mathrm{P}>\mathrm{S}$, o que reflete as exigências da planta.

Constatou-se interação significativa entre os tratamentos e as épocas de avaliação na determinação indireta da concentração de clorofila, o que não ocorreu no tratamento deficiente em N (Figura 2). Houve diferença entre os tratamentos completo (40,14 unidades SPAD) e com deficiência em N (34,72 unidades SPAD), na média das épocas de avaliação. No material colhido aos 35 dias após o início dos tratamentos, ocorreu correlação significativa entre a atividade enzimática da redutase do nitrato $\left(\mathrm{RNO}_{3}{ }^{-}\right.$, expressa em $\mu \mathrm{g}$ de $\left.\mathrm{NO}_{2}{ }^{-} \mathrm{g}^{-1} \mathrm{~h}^{-1}\right)$ e a determinação indireta da concentração de clorofila, em valores SPAD, nos tratamentos completo e com deficiência em $\mathrm{N}\left(\mathrm{RNO}_{3}{ }^{-}=-29,44+0,95\right.$ SPAD, $\mathrm{r}=0,81$, $\mathrm{P}<0,05)$, correspondendo às atividades de 9,43 e $3,02 \mu \mathrm{g}$ de $\mathrm{NO}_{2}^{-} \mathrm{g}^{-1} \mathrm{~h}^{-1}$, respectivamente, para os valores SPAD de 40,91 e 34,17. O uso do clorofilômetro seria, pois, alternativa na avaliação do estado nutricional do nitrogênio.

Entretanto, as deficiências dos outros macronutrientes também influenciaram as leituras. Por causa disso, a determinação indireta da clorofila como um meio de avaliar o estado nutricional é questionável: a clorose pode 
ser causada pela deficiência de vários nutrientes envolvidos na biossíntese e na composição da clorofila (Shaahan et al., 1999; Mengel \& Kirkby, 2001).

Todos os tratamentos com deficiência apresentaram produção de massa de matéria seca total inferior à do completo, o $\mathrm{N}$ foi o nutriente que mais limitou o crescimento (Tabela 3). Na condição de deficiência de N, a produção de massa de matéria seca total foi reduzida em até $68 \%$ em relação ao tratamento completo, afetando principalmente, a parte aérea. Nos tratamentos

Tabela 3. Produção média de matéria seca $\left(\mathrm{g}_{\text {planta }}{ }^{-1}\right)$ da raíz, parte aérea e total da mamoneira, cv. Iris, desenvolvida em soluções nutritivas completa e deficientes em N, P, K, Ca, Mg e $S^{(1)}$.

\begin{tabular}{lccc}
\hline Tratamento & Raiz & Parte aérea & Total \\
\hline Completo & $11,4 \mathrm{a}$ & $18,4 \mathrm{a}$ & $29,7 \mathrm{a}$ \\
Completo sem N & $4,7 \mathrm{c}$ & $5,0 \mathrm{c}$ & $9,6 \mathrm{~d}$ \\
Completo sem P & $11,1 \mathrm{a}$ & $16,4 \mathrm{ab}$ & $27,5 \mathrm{ab}$ \\
Completo sem K & $9,1 \mathrm{ab}$ & $15,3 \mathrm{ab}$ & $24,4 \mathrm{abc}$ \\
Completo sem Ca & $6,2 \mathrm{bc}$ & $8,9 \mathrm{bc}$ & $15,1 \mathrm{~cd}$ \\
Completo sem Mg & $7,2 \mathrm{bc}$ & $12,0 \mathrm{abc}$ & $19,2 \mathrm{bc}$ \\
Completo sem S & $6,7 \mathrm{bc}$ & $11,0 \mathrm{abc}$ & $17,6 \mathrm{~cd}$ \\
\hline $\mathrm{CV}(\%)$ & 13,39 & 21,60 & 16,20
\end{tabular}

${ }^{(1)}$ Médias seguidas por letras distintas, na coluna, diferem entre si pelo teste de Tukey a $5 \%$ de probabilidade. com deficiências em $\mathrm{Ca}$, $\mathrm{S}$ e $\mathrm{Mg}$, as produções de massa de matéria seca foram $49 \%, 41 \%$ e $35 \%$ menores, respectivamente, em relação à produção da massa de matéria seca total obtida no tratamento completo. Os tratamentos deficientes em $\mathrm{Pe} \mathrm{K}$ foram os que apresentaram os menores efeitos, possivelmente por causa da acumulação anterior ao início da omissão.

A relação entre a concentração de $\mathrm{Ne}$ Ca foi significativa em todas as partes da mamoneira e produção de massa de matéria seca total na ocasião da colheita das plantas, bem como, na relação entre a concentração de Mg nos limbos e pecíolos superiores e inferiores e produção de massa de matéria seca total (Tabela 4). As relações entre a concentração de $\mathrm{N}$ nas partes da planta e a produção de massa de matéria seca ajustaram-se ao modelo de equação de regressão quadrática, o que permitiu calcular a concentração crítica no limbo superior, onde ocorreu maior concentração do nutriente (46,7 $\left.\mathrm{g} \mathrm{kg}^{-1}\right)$. Quanto ao $\mathrm{Ca}$ e $\mathrm{Mg}$, as relações entre as concentrações nos tecidos e a produção total de matéria seca também se ajustaram às equações quadráticas. A concentração crítica de Ca no limbo da folha superior foi de 13,8 $\mathrm{g} \mathrm{kg}^{-1}$; entretanto para o $\mathrm{Mg}$, o pecíolo da folha inferior apresentou maior correlação entre o teor e a produção total de matéria seca, correspondendo à concentração crítica de $6,5 \mathrm{~g} \mathrm{~kg}^{-1}$.

Tabela 4. Produção de matéria seca total (Y) da mamoneira, cv. Iris, desenvolvida em solução nutritiva completa e com deficiência em N (Def-N), P (Def-P), K (Def-K), Ca (Def-Ca), Mg (Def-Mg) e S (Def-S), em função da concentração dos nutrientes nas diferentes partes da planta $(\mathrm{X})$.

\begin{tabular}{|c|c|c|c|c|}
\hline Parte da planta & Def-N & $\mathrm{r}$ & Def-P & $\mathrm{r}$ \\
\hline Limbo superior & $Y=-2,42+0,14 X-0,01 X^{2}$ & $0,87 * *$ & $Y=92,60-34,26 X+4,43 X^{2}$ & $0,53^{\mathrm{ns}}$ \\
\hline Pecíolo superior & $Y=62,85-7,11 X+0,24 X^{2}$ & $0,93^{*}$ & $Y=45,29-12,31 X+1,53 X^{2}$ & $0,47^{\mathrm{ns}}$ \\
\hline Limbo inferior & $Y=34,77-3,67 X+0,13 X^{2}$ & $0,87 * *$ & $Y=41,99-14,10 X+2,55 X^{2}$ & $0,62^{\mathrm{ns}}$ \\
\hline Pecíolo inferior & $Y=-26,51+5,39 X-0,13 X^{2}$ & $0,77 *$ & $Y=23,50+4,64 X-0,73 X^{2}$ & $0,38^{\mathrm{ns}}$ \\
\hline Caule & $Y=13,02-1,88 X+0,21 X^{2}$ & $0,72 *$ & $Y=38,79-16,37 X+5,09 X^{2}$ & $0,51^{\mathrm{ns}}$ \\
\hline \multirow[t]{2}{*}{ Raiz } & $Y=-4,00+1,99 X-0,02 X^{2}$ & $0,98 * * *$ & $Y=43,65-12,13 X+1,31 X^{2}$ & $0,45^{\mathrm{ns}}$ \\
\hline & Def-K & & Def-Ca & \\
\hline Limbo superior & $Y=10,82+1,20 X-0,02 X^{2}$ & $0,60^{\text {ns }}$ & $Y=1,34+13,46 X-0,84 X^{2}$ & $0,90 * *$ \\
\hline Pecíolo superior & $Y=-38,99+9,48 X-0,26 X^{2}$ & $0,58^{\text {ns }}$ & $Y=13,46+1,53 X+0,07 X^{2}$ & $0,81^{*}$ \\
\hline Limbo inferior & $\mathrm{Y}=32,31-1,25 \mathrm{X}+0,04 \mathrm{X}^{2}$ & $0,64^{\mathrm{ns}}$ & $Y=9,66+1,55 X-0,02 X^{2}$ & $0,84 * *$ \\
\hline Pecíolo inferior & $Y=23,76+0,16 X+0,003 X^{2}$ & $0,63^{\text {ns }}$ & $Y=18,86-1,48 X+0,16 X^{2}$ & $0,91 * *$ \\
\hline Caule & $Y=32,20-3,53 X+0,30 X^{2}$ & $0,61^{\mathrm{ns}}$ & $-(1)$ & - \\
\hline \multirow[t]{2}{*}{ Raiz } & $Y=29,76-1,11 X+0,04 X^{2}$ & $0,64^{\mathrm{ns}}$ & $\mathrm{Y}=2,20+12,77 \mathrm{X}-1,15 \mathrm{X}^{2}$ & $0,87 * *$ \\
\hline & Def-Mg & & Def-S & \\
\hline Limbo superior & $\mathrm{Y}=3,13+4,67 \mathrm{X}-0,13 \mathrm{X}^{2}$ & $0,75^{*}$ & $Y=244,8-178,9 X+34,55 X^{2}$ & $0,55^{\mathrm{ns}}$ \\
\hline Pecíolo superior & $Y=19,84-0,76 X+0,21 X^{2}$ & $0,91 * *$ & $Y=-1,55+24,32 X-4,41 X^{2}$ & $0,50^{\mathrm{ns}}$ \\
\hline Limbo inferior & $Y=-12,30+13,64 X-1,05 X^{2}$ & $0,90 * *$ & $Y=-19,65+19,03 X-1,96 X^{2}$ & $0,50^{\text {ns }}$ \\
\hline Pecíolo inferior & $Y=22,79-2,83 X+0,53 X^{2}$ & $0,95 * * *$ & $Y=180-191,27 X+56,10 X^{2}$ & $-0,66^{\mathrm{ns}}$ \\
\hline Caule & $Y=23,92+1,95 X-0,26 X^{2}$ & $-0,35^{\mathrm{ns}}$ & $Y=-50,82+178,1 X-9,36 X^{2}$ & $0,46^{\mathrm{ns}}$ \\
\hline Raiz & $Y=1330-390,5 X+29,06 X^{2}$ & $0,10^{\text {ns }}$ & $Y=-11,28+25,00 X-4,14 X^{2}$ & $-0,40^{\mathrm{ns}}$ \\
\hline
\end{tabular}

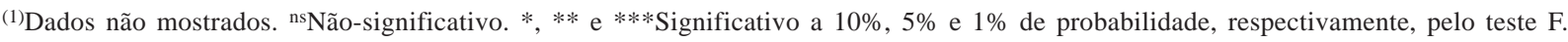




\section{Conclusões}

1. O N e o K são os primeiros elementos a apresentar sintomas visuais de deficiência, seguidos por $\mathrm{Ca}, \mathrm{Mg}$, $\mathrm{S}$ e $\mathrm{P}$.

2. O limbo da folha superior é a parte da planta que melhor reflete o estado nutricional da mamoneira para $\mathrm{N}$ e Ca, e o pecíolo da folha inferior, para $\mathrm{Mg}$.

3. O clorofilômetro pode ser utilizado na avaliação do estado nutricional da mamoneira para $\mathrm{N}$, em qualquer época de crescimento, desde quando for esse o elemento limitante.

4. A produção total de matéria seca das plantas é afetada pelas deficiências dos macronutrientes e, o N é o mais limitante.

5. As determinações da atividade da redutase do nitrato, de teores solúveis de $\mathrm{N}\left(\mathrm{NO}_{3}^{-}\right), \mathrm{P}\left(\mathrm{H}_{2} \mathrm{PO}_{4}^{-}\right)$e $\mathrm{K}^{+}$ e os testes rápidos podem ser utilizados na diagnose do estado nutricional da mamoneira.

\section{Agradecimentos}

Ao $\mathrm{CNPq}$, pela bolsa concedida a Eurípedes Malavolta; a Cristiano Elemar Voll, aluno de Pós-graduação em Produção Vegetal - USP/Esalq, pela ajuda na edição das fotografias; ao Grupo Itaquere (Primavera do Leste, MT), pelo fornecimento das sementes da mamoneira cv. Iris.

\section{Referências}

BELTRÃO, N.E. de M.; SILVA, L.C.; MELO, F.B. Mamona consorciada com feijão visando produção de biodiesel, emprego e renda. Bahia Agrícola, v.5, p.34-37, 2002.

BELTRÃO, N.E. de M.; SILVA, L.C.; VASCONCELOS, O.L.; AZEVEDO, D.M.P. de; VIEIRA, D.J. Fitologia. In: AZEVEDO, D.M.P.; LIMA, E.L. (Ed.). O agronegócio da mamona no Brasil, Brasília: Embrapa Informação Tecnológica, 2001. p.37-61.

CANECCHIO FILHO, V.; FREIRE, E.S. Adubação da mamoneira. I: Experiências preliminares. Bragantia, v.17, p.243-259, 1958.

CANECCHIO FILHO, V.; ROCHA, J.L.V.; FREIRE, E.S. Adubação da mamoneira. III: Experiências com doses crescentes de nitrogênio, fósforo e potássio. Bragantia, v.22, p.765-775, 1963.

EPSTEIN, E. Nutrição mineral de plantas: princípios e perspectivas. Rio de Janeiro: Livros Técnicos e Científicos, 1975. 341p.

FERNANDES, C.S. Análises de tecidos vegetais por meio de "spot tests". Pesquisa Agropecuária Brasileira, v.2, p.237-244, 1967.
FREIRE, R.M.M. Ricinoquímica. In: AZEVEDO, D.M. de P.; LIMA, E.L. (Ed.). O agronegócio da mamona no Brasil, Brasília: Embrapa Informação Tecnológica, 2001. p.295-335.

HOCKING, P.J. Accumulation and distribution of nutrients in fruits of castor bean (Ricinus communis L.). Annals of Botany, v.49, p.5162, 1982.

IBGE. Banco de dados agregados. Disponível em: <http:www.sidra.ibge.gov.br> Acesso em: 9 jul. 2003.

JOHNSON, C.M.; STOUT, P.R.; BROYER, T.C.; CARLTON, A.B. Comparative chlorine requirements of different plants species. Plant and Soil, v.8, p.337-353, 1957.

MALAVOLTA, E. Pruebas rápidas en tejidos vegetales. In: SARASSOLA, A.A.; SARASSOLA, M.A.R. (Ed.). Fitopatologia - Curso moderno. Buenos Aires: Editora Hemisferio Sur, 1975. p.260-262.

MALAVOLTA, E.; VITTI, G.C.; OLIVEIRA, S.A. Avaliação do estado nutricional das plantas: princípios e aplicações. 2.ed. Piracicaba: Potafos, 1997. 319p.

MENGEL, K.; KIRKBY, E. Principles of plant nutrition. 5.ed. Dordrecht/Boston/London: Kluwer Academic Publishers, 2001. 849p. MINOLTA CAMERA Co. Manual for chlorophyll meter Spad502. Osaka: Minolta, Radiometric Instruments Divisions, 1989. 22p. MULDER, E.G.; BOXMA, R.; VAN VENN, W.L. The effect of molybdenum and nitrogen deficiencies on nitrate reduction in plant tissue. Plant and Soil, v.10, p.335-355, 1959.

NAKAGAWA, J.; NEPTUNE; A.M.L.; MURAOKA, T. Absorção e translocação de fósforo em dois cultivares de mamoneira (Ricinus communis L.), "Campinas" e "Guarani". Anais da Escola Superior de Agricultura "Luiz de Queiroz", v.39, p.319-335, 1982.

ROCHA, J.L.V.; CANECCHIO FILHO, V.; FREIRE, E.S. Adubação da mamoneira. V: Experiência com vários fosfatos em solo do arenito Botucatu. Bragantia, v.23, p.291-297, 1964.

ROJAS, A.I.; NEPTUNE, A.M.L. Efeitos dos macronutrientes e do ferro no crescimento e composição química da mamoneira (Ricinus communis L.) cultivada em solução de nutrientes. Anais da Escola Superior de Agricultura “Luiz de Queiroz”, v.28, p.31-67, 1971. SAS INSTITUTE (Cary, United States). SAS/STAT user's guide: version 6.11. 4.ed. Cary, 1996. v.2. 842p.

SHAAHAN, M.M.; EL-SAYED, A.A.; ABOU EL-NOUR, E.A.A. Predicting nitrogen, magnesium and iron nutritional status in some perennial crops using a portable chlorophyll meter. Scientia Horticulturae, v.82, p.339-348, 1999.

ULRICH, A. Plant analysis - methods and interpretation of results. In: KITCHEN, H.B. (Ed.). Diagnostic techniques for soils and crops. Washington: The America Potash Institute, 1948. p.157-198. ULRICH, A.; HILLS, E.J. Plant analysis as an aid in fertilizing sugar crops: part I. Sugar beets. Principles and practices of plant analysis. In: WALSH, L.M.; BEATON, J.D. (Ed.). Soil testing and plant analysis. Madison: Soil Science Society of America, 1973. p.271-288.

VELOSO, C.A.C.; MURAOKA, T.; MALAVOLTA, E.; CARVALHO, J.G. de. Diagnose de deficiências de macronutrientes em pimenta-do-reino. Pesquisa Agropecuária Brasileira, v.33, p.1889-1896, 1998.

Recebido em 2 de março de 2004 e aprovado em 15 de setembro de 2004 